Phonon damping in the simultaneous presence of intense radiation and magnetic fields

This content has been downloaded from IOPscience. Please scroll down to see the full text.

1976 J. Phys. C: Solid State Phys. 92971

(http://iopscience.iop.org/0022-3719/9/15/020)

View the table of contents for this issue, or go to the journal homepage for more

Download details:

IP Address: 150.163.34.14

This content was downloaded on 25/08/2015 at 19:35

Please note that terms and conditions apply. 


\title{
Phonon damping in the simultaneous presence of intense radiation and magnetic fields
}

\author{
L C M Miranda \\ Departmento de Física, Universidade de Brasilia, 70.000 Brasília, DF, Brazil
}

Received 9 January 1976, in final form 16 March 1976

\begin{abstract}
Phonon scattering by electrons in the simultaneous presence of intense laser and magnetic fields is discussed. A kinetic equation is derived, and the rate of change of the phonon population is calculated. For laser radiation propagating parallel to the magnetic field, it is found that multiphoton processes are dominant when the laser frequency is near the electron cyclotron frequency. Furthermore, the damping rate is found to decrease as the. laser frequency approaches the electron cyclotron frequency.
\end{abstract}

\section{Introduction}

There has been recent interest in the study of the interaction of intense laser fields with semiconductors (Ephstein 1970, 1971, Puchkov and Ephstein 1974, Bass and Granovskii 1971) and plasmas (Seely and Harris 1973, Cohn et al 1972), aiming not only at the excitation of higher harmonics and amplification of high-frequency phonons, but also to be able to change the conductivity in these systems. Since the laser frequency is usually much greater than the electron cyclotron frequency, an external magnetic field probably has little effect on the rate of absorption of laser energy by the system, but has a major effect on the confinement of the electron states. However, a resonance condition, where the laser frequency is equal to the electron cyclotron frequency, may be approached either by increasing the magnetic field strength or by using longer-wavelength lasers. Intense submillimeter lasers are becoming available (Lax and Cohn 1973) and it is therefore important to consider the cylotron resonance absorption of this radiation. In this paper we consider phonon scattering by electrons in the field of an intense laser radiation (Ephstein 1970) and include the effects of a strong magnetic field. We assume the ion cyclotron frequency to be much smaller than the phonon frequency so that the phonons are affected by the magnetic field only indirectly through the electrons. The laser beam is treated as a classical plane electromagnetic wave in the dipole approximation. The electron states are described by the solution to the Schrödinger equation for an electron in the laser field and a uniform static magnetic field.

Phonon scattering by the electrons is treated using first-order perturbation theory retaining, however, the laser field strength to all orders. The transition probabilities are used to write a kinetic equation for the phonon population (Harris 1969), from which the damping rate is obtained. For the case of laser radiation propagating parallel to the magnetic field, the phonon damping is found to decrease as the laser frequency approaches the electron cyclotron frequency. 


\section{Formulation}

Let the magnetic field be in the $+z$ direction. Neglecting the spatial dependence of the electromagnetic field of the laser beam, the vector potential of the laser and magnetic field is thus taken to be

$$
\begin{aligned}
& A(y, t)=A(t)-B y e \\
& A(t)=\left(c E_{0} / \omega\right)\left[e_{x} \cos (\omega t)+e_{y} \sin (\omega t)\right]
\end{aligned}
$$

where $B$ is the magnetic field strength and $A(t)$ represents the field of the laser beam, assumed to be a right-hand circularly polarized plane wave propagating parallel to the $z$ axis. The solution to the time-dependent Schrödinger equation for the electron motion is (Seely 1974)

$$
\psi=L^{-1} \exp (\mathrm{ip} \cdot \boldsymbol{x} / \hbar) \exp \left(-\mathrm{i} E_{n} t / \hbar\right) \exp \left(-\frac{\mathrm{i}}{2 m h} \int^{t} R\left(t^{\prime}\right) \mathrm{d} t^{\prime}\right) \phi_{n}(\zeta)
$$

where

$$
\begin{aligned}
& E_{n}=\hbar \omega_{c}\left(n+\frac{1}{2}\right), \quad n=0,1,2, \ldots, \\
& p=\left(p_{x}, Q(t), p_{z}\right), \\
& R(t)=[p-(e / c) A(t)]^{2}-\left[p_{x}-G(t)\right]^{2} \\
& \phi_{n}(\zeta)=\left(m \omega_{d} / \Pi \hbar\right)^{1 / 4}\left(2^{n} n !\right)^{-1 / 2} \exp \left(-\zeta^{2} / 2\right) H_{n}(\zeta), \\
& \zeta=\left(m \omega_{c} / \hbar\right)^{1 / 2} y-\left(m \omega_{c} \hbar\right)^{-1 / 2}\left[p_{x}-G(t)\right] .
\end{aligned}
$$

Here $\omega_{\mathrm{c}}=|e| \mathrm{B} / \mathrm{mc}$ is the cyclotron frequency, $p_{x}$ and $p_{z}$ are constants of motion, $n$ is the Landau-level quantum number, and $\phi_{n}(\zeta)$ is the harmonic-oscillator wavefunction. The real functions of time $G(t)$ and $Q(t)$ are determined by the equation (Seely 1974)

$$
G(t)+\mathrm{i} Q(t)=\frac{e \omega_{c}}{c} \int^{t} \mathrm{~d} t^{\prime}\left[A_{y}\left(t^{\prime}\right)-\mathrm{i} A_{x}\left(t^{\prime}\right)\right] \exp \left[\mathrm{i} \omega_{c}\left(t-t^{\prime}\right)\right],
$$

where $A_{x}(t)$ and $A_{y}(t)$ are components of $A(t)$. We refer the reader to Seely's paper (1974) for a more detailed discussion of the solution (2).

Treating the electron-phonon interaction as a perturbation, the probability amplitude for the transition from state $1\left(n_{1}, p_{1 x}, p_{1 z}\right)$ to state $2\left(n_{2}, p_{2 x}, p_{2 z}\right)$ due to the collision with a phonon of momentum $\hbar \boldsymbol{k}$ is

$$
a(1 \rightarrow 2 ; \boldsymbol{k})=-\frac{\mathrm{i}}{\frac{1}{h}} \iint \mathrm{d}^{3} \times \mathrm{d} t \psi_{2}^{*} v_{k} \exp \left[\mathrm{i}\left(\boldsymbol{k} \cdot \boldsymbol{x}-\omega_{k} t\right)\right] \psi_{1}
$$

where $v_{k}$ is the electron-phonon coupling. Substituting (2) into (6) and performing the integration over $x$ and $z$, we obtain:

$$
\begin{aligned}
a(1 \rightarrow 2 ; \boldsymbol{k})= & -\frac{\mathrm{i} v_{k}}{\hbar} \frac{(2 \pi \hbar)^{2}}{L^{2}} \delta\left(p_{2 x}-p_{1 x}-\hbar k_{x}\right) \delta\left(p_{2 z}-p_{1 z}-\hbar k_{x}\right) \\
& \times \int \mathrm{d} t \exp \left(\frac{\mathrm{i}}{\hbar}\left(E_{n_{2}}-E_{n_{1}}-\hbar \omega_{k}\right)+\frac{\mathrm{i}}{2 m \hbar} \int^{t} \mathrm{~d} t^{\prime}\left(R_{2}-R_{1}\right)\right)_{I(t)},
\end{aligned}
$$


where

$$
\begin{aligned}
& I(t)=\int_{-\infty}^{+\infty} \mathrm{d} y \exp \left(i k_{y} y\right) \phi_{n_{2}}\left(\zeta_{2}\right) \phi_{n_{1}}\left(\zeta_{1}\right) \\
& \zeta_{i}=\left(m \omega_{\mathrm{c}} / \hbar\right)^{1 / 2} y-\left(m \omega_{\mathrm{c}} \hbar\right)^{-1 / 2}\left[p_{i x}-G(t)\right], \quad i=1 \text { or } 2 .
\end{aligned}
$$

Integrals similar to the one in (6) have been considered by several authors (Lee and Tzoar 1969, Gomes and Miranda 1975). The result is (Gomes and Miranda 1975)

$$
I(t)=\exp \left\{i k_{y}\left[p_{1 x}+p_{2 x}-2 G(t)\right] / 2 m \omega_{c}\right\} \nsucc\left(n_{2}, n_{1}, \rho\right)
$$

where $\chi\left(n_{2}, n_{1}, \rho\right)$ has been defined previously (Gomes and Miranda 1975) with $\rho=\hbar k_{\perp}^{2} /$ $2 m \omega_{\mathrm{c}}$.

The integration over $t$ in (5) may be performed after expanding the factors that are periodic in time in the Fourier series:

$$
\sum_{v=-\infty}^{+\infty} F_{v}(\boldsymbol{k}) \exp (-\mathrm{i} v \omega t)=\exp \left[\frac{\mathrm{i} k_{x}}{m} \int^{t} \mathrm{~d} t^{\prime}\left(G-\frac{e}{c} A_{x}\right)-\frac{\mathrm{i} k_{y} G}{m \omega_{\mathrm{c}}}-\frac{\mathrm{i} e k_{z}}{m c} \int^{t} \mathrm{~d} t^{\prime} A_{z}\right] \text {. }
$$

Then (5) may be written as

$$
\begin{aligned}
a(1 \rightarrow 2 ; \boldsymbol{k})= & -\frac{\mathrm{i} v_{k}}{\hbar} \frac{(2 \pi \hbar)^{3}}{L^{2}} \delta\left(p_{2 x}-p_{1 x}-\hbar k_{x}\right) \delta\left(p_{2 z}-p_{1 z}-\hbar k_{z}\right) \\
& \times \exp \left[i k_{y}\left(p_{1 x}+p_{2 x}\right) / 2 m \omega_{\mathrm{c}}\right] \chi\left(n_{2}, n_{1}, \rho\right) \sum_{v=-\infty}^{+\infty} F_{v}(\boldsymbol{k}) \\
& \times \delta\left(E_{n_{2}}+\frac{p_{2 z}^{2}}{2 m}-E_{n_{1}}-\frac{p_{1 z}^{2}}{2 m}-\hbar \omega_{k}-v \hbar \omega\right) .
\end{aligned}
$$

From the well known relation between the scattering amplitude and the T-matrix (Roman 1965) we can then use (9) to obtain the transition probability per unit time, $T_{v}(1 \rightarrow 2, k)$, for the transition from state 1 to state 2 due to a collision with a phonon $\boldsymbol{k}$ with absorption $(v>0)$ or emission $(v<0)$ of $|v|$ photons. One gets:

$$
T_{v}(1 \rightarrow 2 ; \boldsymbol{k})=(2 \pi / \hbar)\left|v_{k}\right|^{2}\left|\chi\left(n_{2}, n_{1}, \rho\right)\right|^{2}\left|F_{v}(\boldsymbol{k})\right|^{2} \delta\left(E_{n_{2}}+\frac{p_{2 z}^{2}}{2 m}-E_{n_{1}}-\frac{p_{1 z}^{2}}{2 m}-\hbar \omega_{k}-v \hbar \omega\right)
$$

The rate of change of the number of phonons of wavenumber $k, \mathrm{~d} N_{k} / \mathrm{d} t$, is then given in terms of the transition probability as (Harris 1969, Parry and Turner 1969)

$$
\mathrm{d} N_{k} / \mathrm{d} t=\gamma_{k} N_{k}
$$

where

$$
\begin{aligned}
& \gamma_{k}=\sum_{n, p_{x}, p_{z}} \sum_{l=-\infty}^{+\infty} \sum_{v=-\infty}^{+\infty}(2 \pi / \hbar)\left|v_{k}\right|^{2}|\chi(n+l, n, \rho)|^{2} J_{v}^{2}(\lambda / \hbar \omega) \\
& \times\left[f\left(E^{\prime}\right)-f(E)\right] \delta\left(E^{\prime}-E-\hbar \omega_{k}-v \hbar \omega\right) .
\end{aligned}
$$

Here $E^{\prime}=\hbar \omega_{c}\left(n+l+\frac{1}{2}\right)+\left(p_{z}+\hbar k_{z}\right)^{2} / 2 m, E=\hbar \omega_{c}\left(n+\frac{1}{2}\right)+p_{z}^{2} / 2 m,\left|F_{v}(\boldsymbol{k})\right|^{2}=J_{v}^{2}(\lambda / \hbar \omega)$ where $J_{v}$ is the Bessel function of order $v$, and $\lambda=e \hbar k_{\perp} E_{0} / m\left(\omega-\omega_{c}\right)=\hbar k_{\perp} v_{0}$ is the field parameter. In the case of a right-hand circularly polarized plane wave propagating parallel to the magnetic field, the Fourier coefficients $F_{v}(\boldsymbol{k})$ are Bessel functions (Seely 1974). 
In the following we assume a Maxwellian distribution for the electrons. This assumption is relevant to a significant range of semiconductor materials and is valid provided the electron heating in the radiation field may be neglected. The latter is valid if $e^{2} E_{0}^{2}$ $2 m \omega^{2}\left\langle\langle E\rangle\right.$ where $\langle E\rangle=k_{\mathrm{B}} T$ is the average energy of an electron in the absence of the radiation field. Therefore our results are restricted to radiation fields $E_{0}$ smaller than the threshold heating field

$$
E_{\mathrm{h}}=\left(2 m \omega^{2} k_{\mathrm{B}} T / e^{2}\right)^{1 / 2} .
$$

Furthermore, we shall consider only the laser-cyclotron resonance case, namely, $\omega \sim \omega_{\mathrm{c}}$. Then $\lambda \gg \hbar \omega$ and the argument of the Bessel function is large.

For large values of argument, the Bessel function is small except when the order $v$ is equal to the argument. The sum over $v$ in (12) may then be written approximately as

$$
\sum_{v=-\infty}^{+\infty}\left|J_{v}(\lambda / \hbar \omega)\right|^{2} \delta(\Omega-v \hbar \omega)=\frac{1}{2}[\delta(\Omega-\lambda)+\delta(\Omega+\lambda)] .
$$

The factor $\frac{1}{2}$ may be verified by integrating both sides of the equation over $\Omega=E^{\prime}-E$ $-\hbar \omega_{k}$. The first $\delta$-function corresponds to the emission and the second to the absorption of $\lambda / \hbar \omega$ photons. Since $\lambda \gg \hbar \omega$, only multiphoton processes are significant. The phonon damping then becomes

$$
\begin{aligned}
& \gamma_{k}=\frac{\pi}{\hbar} \sum_{n, p_{x}, p_{z}} \sum_{l=-\infty}^{+\infty} v_{k}^{2}|\chi(n+l, n, \rho)|^{2}\left[f(E)\left\{\exp \left[-\left(\lambda+\hbar \omega_{k}\right) / k_{\mathrm{B}} T\right]-1\right\}\right. \\
& \left.\delta\left(E^{\prime}-E-\hbar \omega_{k}-\lambda\right)+f(E)\left\{\exp \left[\left(\lambda-\hbar \omega_{k}\right) / k_{\mathrm{B}} T\right]-1\right\} \delta\left(E^{\prime}-E-\hbar \omega_{k}+\lambda\right)\right] .
\end{aligned}
$$

If we further assume that $\lambda \gg k_{\mathrm{B}} T$ for $\omega$ near $\omega_{\mathrm{c}}$, the contribution of processes in which photons are emitted is negligible compared with the contribution of processes in which photons are absorbed. Under these circumstances, (13) reduces to

$\gamma_{k}=\frac{\pi}{\hbar} \sum_{l=-\infty}^{+\infty} \sum_{n, p_{x}, p_{z}} v_{k}^{2}|\chi(n+l, n, \rho)|^{2} f(E)\left\{\exp \left[\left(\lambda-\hbar \omega_{k}\right) / k_{\mathrm{B}} T\right]-1\right\} \delta\left(E^{\prime}-E-\hbar \omega_{k}+\lambda\right)$.

Equation (14) is quite general and valid for any radiation and magnetic field strengths. The only assumptions are that $\omega$ is near $\omega_{c}$ and that the electrons obey a Maxwellian distribution. Since the existence in most semiconductors of well defined Landau levels is not easily attainable with relatively low magnetic fields, we shall in the following take the classical limit of (14) by letting (Harris 1969, Walters and Harris 1968)

$$
\hbar \rightarrow 0 \quad \text { and } n \rightarrow \infty \text {, }
$$

such that

$$
n \hbar \omega_{\mathrm{c}} \rightarrow \frac{1}{2} m v_{\perp}^{2}
$$

and

$$
\sum_{n, p_{x}, p_{z}}(\ldots) f\left(E_{n p_{x p} p_{z}}\right) \rightarrow \int \mathrm{d}^{3} v(\ldots) f(v)
$$

Hence, expanding (14) in powers of $\hbar$ and retaining only the lowest-order terms, one gets

$$
\gamma_{k}=\frac{\pi v V_{k}^{2}}{\hbar k_{\mathrm{B}} T}\left(k_{\perp} v_{0}-\omega_{k}\right) \sum_{l=-\infty}^{+\infty} \int \mathrm{d}^{3} v J_{l}^{2}\left(k_{\perp} v_{\perp} / \omega_{\mathrm{c}}\right) f(\boldsymbol{v}) \delta\left(l \omega_{\mathrm{c}}+k_{z} v_{z}-\omega_{k}+k_{\perp} v_{0}\right)
$$


where we have written $\lambda$ as $\hbar k_{\perp} v_{0}$ with $v_{0}=e E_{0} / m\left(\omega-\omega_{c}\right)$, and replaced $|\chi(n+l, n, \rho)|^{2}$ by its classical limit (Walters and Harris 1968) $|\chi(n+l, n, \rho)|^{2} \rightarrow J_{l}^{2}\left(k_{\perp} v_{\perp} / \omega_{c}\right)$. One notices that $v_{k}^{2}$ is proportional to $\hbar$ so that (18) is in fact independent of $\hbar$.

For $E_{0}=0\left(v_{0}=0\right),(18)$ reduces to the well known expression for the phonon damping in a uniform magnetic field in the classical limit (Harris 1969), On the other hand, for $B \rightarrow 0\left(\omega_{c} \rightarrow 0\right)$, the argument of the Bessel functions in (18) is large, so that, as before, we may approximate $\gamma_{k}$ by

$$
\gamma_{k} \underset{(B \rightarrow 0)}{\rightarrow} \frac{2 \pi V v_{k}^{2}}{\hbar k_{\mathrm{B}} T}\left(k_{\perp} v_{0}-\omega_{k}\right) \int \mathrm{d}^{3} v f(\boldsymbol{v}) \delta\left(\boldsymbol{k} \cdot \boldsymbol{v}-\omega_{k}+k_{\perp} v_{0}\right) .
$$

This is essentially the same result as one would get for the phonon damping if we had only the radiation field, in the limit of either $E_{0}$ very large or $\omega$ very small. It can easily be worked out using the electron wavefunction in the presence of the radiation field only (Seely and Harris 1973).

Going back to (18), substituting $f(v)$ by $n_{0}\left(\pi v_{\mathrm{T}}^{3}\right)^{-3 / 2} \exp \left(-v^{2} / v_{\mathrm{T}}^{2}\right)$, where $v_{\mathrm{T}}^{2}=2 k_{\mathrm{B}} T / m$, and performing the integration over $v_{z}$ using the $\delta$-function, one has

$$
\gamma_{k}=\frac{2 \pi^{1 / 2} n_{0} V v_{k}^{2}}{\hbar m v_{\mathrm{T}}^{2}}\left(\frac{k_{\perp} v_{0}-\omega_{k}}{\left|k_{z}\right| v_{\mathrm{T}}}\right) \sum_{l=-\infty}^{+\infty} \exp \left[-\left(k_{\perp} v_{0}+l \omega_{\mathrm{c}}-\omega_{k}\right)^{2} / k_{z}^{2} v_{\mathrm{T}}^{2}\right] F_{l}\left(k_{\perp} v_{\mathrm{T}} / \omega_{\mathrm{c}}\right)
$$

where

$$
F_{l}\left(k_{\perp} v_{\mathrm{T}} / \omega_{\mathrm{c}}\right)=\int_{0}^{+\infty} \mathrm{d} x \exp (-x) J_{l}^{2}\left(k_{\perp} v_{\mathrm{T}} \sqrt{x} / \omega_{\mathrm{c}}\right)=\exp \left[-k_{\perp}^{2} v_{\mathrm{T}}^{2} / 2 \omega_{\mathrm{c}}^{2}\right] I_{l}\left(k_{\perp}^{2} v_{\mathrm{T}}^{2} / 2 \omega_{\mathrm{c}}^{2}\right)
$$

Equation (20) is the expression for $\gamma_{k}$ which we wish to discuss. The first point one notices is that, regardless of the value of $E_{0}$, for $\omega=\omega_{\mathrm{c}}$ (i.e. $\left.v_{0} \rightarrow \infty\right) \gamma_{k}$ vanishes. Physically this result may be understood as follows. Consider the problem of a single electron in an electromagnetic field described by $\boldsymbol{A}(t)$ and moving in a potential $V$ (the lattice potential), For simplicity we shall neglect the external magnetic field. We have

$$
H=[p-(e / c) A(t)]^{2} / 2 m+V=H_{0}+V .
$$

In the case of a strong field, such that $|e A| \gg|V|$, the above Hamiltonian may be approximated by the first term $H_{0}$ which is the free-particle Hamiltonian. In other words, under strong fields, the electron no longer sees the phonons; the electron-phonon interaction becomes frozen $\left(\gamma_{k}=0\right)$. Hence the fact that $\gamma_{k}$ vanishes for $\omega=\omega_{c}$ may be understood as if, at the resonance, the radiation field were infinitely large.

The second point regarding (20) is that for $\omega$ near $\omega_{\mathrm{c}}$, but not necessarily at resonance, the phonon population may in principle grow with time for wavevectors $\boldsymbol{k}$ in a general direction $\theta$, provided $k_{\perp} v_{0}>\omega_{k}$ or $v_{0}>v_{\mathrm{s}}$, the sound velocity. This condition is in fact amply satisfied for $\omega \sim \omega_{\mathrm{c}}$. However, the estimative of this growth rate from $(20)$ is quite tedious. Nevertheless, one may get the order of magnitude of the size of this effect by considering the case where $k_{\perp} v_{\mathrm{T}} \ll \omega_{\mathrm{c}}$. In this case, the $l=0$ component in (20) and (21) is the dominant one and we have

$\gamma_{k} \simeq \frac{2 \pi^{1 / 2} n_{0} V v_{k}^{2}}{\hbar m v_{\mathrm{T}}^{2}} \frac{\left(k_{\perp} v_{0}-\omega_{k}\right)}{\left|k_{z}\right| v_{\mathrm{T}}} \exp \left[-\left(k_{\perp} v_{0}-\omega_{k}\right)^{2} / k_{z}^{2} v_{\mathrm{T}}^{2}\right]$

which is maximum for

$$
\left(k_{\perp} v_{0}-\omega_{k}\right) /\left|k_{z}\right| v_{\mathrm{T}}=\frac{1}{2}
$$


Since $v_{0} \gg v_{\mathrm{s}}$, this latter condition is roughly $v_{0}=v_{\mathrm{T}} / 2$, from which one obtains the critical field $E_{\mathrm{c}}=\left[m\left(\omega-\omega_{\mathrm{c}}\right)^{2} k_{\mathrm{B}} T / 2 e^{2}\right]^{1 / 2}$, which maximizes $\gamma_{k}$. One notices that $F_{\mathrm{c}}$ must be smaller than $E_{\mathrm{h}}$ in order that heating may be neglected. By writing $\left|\omega-\omega_{\mathrm{c}}\right|=$ $r \omega_{\mathrm{c}}$, where $r$ measures the matching of the resonance, we have $E_{\mathrm{c}}=\left|\omega-\omega_{\mathrm{c}}\right| E_{\mathrm{h}} / 2 \omega \simeq r E_{\mathrm{h}} / 2$. This means that, in fact, heating may be neglected, as $r<1$ near resonance.

Assuming the jellium model, $v_{k}^{2}=2 \pi e^{2} \hbar \omega_{k} / V k_{\mathrm{D}}^{2}$, where $k_{\mathrm{D}} \simeq \omega_{\mathrm{p}} / v_{\mathrm{T}}$, and taking $E_{0} \simeq E_{\mathrm{c}},(22)$ reduces to

$$
\gamma_{k} \sim \omega_{k}
$$

provided $k<k_{\mathrm{D}}, \omega_{\mathrm{c}} / v_{\mathrm{T}}$. For a typical semiconductor, like n-type InSb, with $m=10^{-29} \mathrm{~g}$, $n_{0}=10^{16} \mathrm{~cm}^{-3}, T=300 \mathrm{~K}$ and $B=10 \mathrm{kG}$, the critical intensity for which (23) is valid, taking $r=0 \cdot 1$, turns out to be of the order of $500 \mathrm{~W} \mathrm{~cm}^{-2}$, at a frequency of about $1.6 \times 10^{13} \mathrm{~s}^{-1}$.

\section{Conclusions}

In this paper we have looked at the effects of the laser-cyclotron resonance on phonon damping in semiconductors. It has been shown that, as one approaches resonance, the damping coefficient vanishes. Physically, this is understood as if at resonance the radiation field were infinitely large, which in turn entails that the electron-phonon interaction becomes frozen.

If, however, one is not at resonance, but near it, it has also been shown that one might in principle observe a growth of the phonon population. The expression (22) for the growth rate is formally analogous to the usual growth rate in a DC electric field where $v_{0}$ replaces $v_{\mathrm{d}}$, the drift velocity. For long-wavelength acoustic phonons, the growth rate near resonance has been shown to be of the order of $\omega_{k}$ for $E_{0}=E_{\mathrm{c}}$. A numerical estimate of the required critical intensity for a typical semiconductor like n-type InSb has also been given.

In any event, although our model contains a number of simplifying assumptions, we believe that, at least qualitatively, the essential conclusions may be useful in explaining future experimental results.

\section{References}

Bass F G and Granovskii M Ya 1971 Sov. Phys.-Solid St. 121948

Cohn D R, Halverson W, Lax B and Chase C E 1972 Phys. Rev. Lett. 291544

Ephstein E M 1970 Sov. Phys.-Solid St. 112213

1971 JETP Lett. 13364

Gomes M A F and Miranda L C M 1975 Phys. Rev. B 123788

Harris E G 1969 in Advances in Plasma Physics Vol 3 eds A Simon and W B Thompson (Reading, Mass: Addison-Wesley)

Lax B and Cohn D R 1973 Appl. Phys. Lett. 23363

Lee Y C and Tzoar N 1969 Phys. Rev. 1781303

Parry W E and Turner R E 1969 Many-Body Problem (New York: Benjamin)

Puchkov V I and Ephstein E M 1974 Sov. Phys.-Semicond. 71254

Roman P 1965 Advanced Quantum Theory (Reading, Mass: Addison-Wesley) p 285

Seely J F 1974 Am. J. Phys. 42326

Seely J F and Harris E G 1973 Phys. Rev. A 71064

Walters G M and Harris E G 1968 Phys. Fluids 11112. 